\title{
Estrategia de Administración en el Área de Salud Pública-Privada
}

\author{
Management Strategy in the Public-Private Health Area \\ Estratégia de Gestão na Área Público-Privada de Saúde
}

\author{
Juan M. Haro-Alvarado I \\ juanmharo@gmal.com \\ Javier I. Haro-Alvarado II \\ javierhaaro@gmail.com \\ Mariela G. Macías-Intriago ${ }^{\text {III }}$ \\ mariela.gm@gmail.com
}

\author{
Nakin A. Veliz-Mero IV \\ nakinaveliz@gmail.com \\ Julissa F. Toala-Sornoza V \\ julissaftoala@gmail.com \\ Tatiana A. Solís-Lino VI \\ tatianaa@gmail.com
}

Correspondencia: juanmharo@gmail.com

I. Doctor en Medicina y Cirugía; Magister en Gerencia en Salud; Especialista en Medicina Interna; Diplomado en Docencia Universitaria; Docente de la Facultad de Ciencias de la Salud, Universidad Técnica de Manabí, Portoviejo, Ecuador.

II. Doctor en Medicina y Cirugía; Especialista en Auditoria Médica; Diplomado en Ciencias de la Educación para la Salud; Magister en Medicina Tropical; Docente de la Facultad de Ciencias de la Salud- Universidad Técnica de Manabí, Portoviejo, Ecuador.

III. Doctor en Medicina y Cirugía; Magister en Epidemiologia; Directora Distrito de Salud Portoviejo; Docente de la Facultad de Ciencias de la Salud - Universidad Técnica de Manabí, Portoviejo, Ecuador.

IV. Doctor en Medicina y Cirugía; Magister en Gestión y Desarrollo Social; Magister en Gerencia en Salud para el Desarrollo Local; Director Distrito Jaramijo - Manta - Montecristi- Salud; Docente de la Universidad Laica Eloy Alfaro de Manabí, Manta, Ecuador.

v. Licenciada en Enfermería; Centro de Salud Andrés de Vera, Portoviejo, Ecuador

VI. Licenciada en Enfermería, Centro de Salud rural Machalilla, Jipijapa, Ecuador. 


\section{Resumen}

El objetivo de este artículo es estudiar las estrategias formuladas, las mismas que ayudan ordenar y a asignar, con base sus condiciones así como identificar sus deficiencias internas, los recursos que requiere para el desarrollo de los procesos de su gestión con lo que persigue lograr un cambio proyectado en la organización acorde con las exigencias demandas o necesidades de los diferentes sectores de la sociedad y de la misma institución, constituyéndose en el componente clave mediante la gestión en busca del cambio con el propósito de lograr un mejor desempeño y mayor eficiencia en la entidad, requisito indispensable para el avance de la institución en el mundo actual. Los sistemas de salud y sus subsistemas (clínicas, hospitales, unidades médicas, etcétera) deben contar con una administración eficiente, con el objeto de poder cumplir las metas que contemplan los diferentes programas de salud, y con ello dar respuesta a las necesidades que la sociedad les demande. Las organizaciones además de depender de la tecnología deben contar con el recurso humano capacitado coherente con las funciones a desempeñar en la actualidad se considera al personal más adecuado que desarrolle su actividad en un espacio eficiente y eficaz talentos que aprovechan la oportunidad en el trabajo para desarrollarse. El Seguro General Obligatorio del IESS tiene tres fuentes de financiamiento: la aportación individual obligatoria de los afiliados, la aportación obligatoria de los empleadores públicos o privados y la contribución del Estado. De igual modo los servicios de salud y prestaciones del Seguro Social Campesino se financian con recursos provenientes del aporte de los empleadores, el aporte de los afiliados al SGO, la contribución obligatoria de seguros públicos y privados, el aporte diferenciado de familias protegidas por el SSC, la contribución del Estado y las asignaciones suplementarias determinadas por el Poder Ejecutivo.

Palabras claves: Estrategia, procesos de gestión, programas de salud, talentos, seguros públicos y privado. 


\begin{abstract}
The objective of this article is to study the strategies formulated, the same ones that help to organize and assign, based on their conditions as well as identify their internal deficiencies, the resources required for the development of the processes of their management with what they seek to achieve a change projected in the organization in accordance with the demands or needs of different sectors of society and the same institution, becoming the key component through management in search of change in order to achieve better performance and greater efficiency in the entity, indispensable requirement for the advancement of the institution in the current world. Health systems and their subsystems (clinics, hospitals, medical units, etc.) must have an efficient administration, in order to meet the goals contemplated by different health programs, and thereby respond to the needs that the society demand them. Organizations as well as relying on technology must have the trained human resources consistent with the functions to be carried out at present. The most appropriate staff is considered to develop their activity in an efficient and effective space talent that take advantage of the opportunity at work to develop. The IESS Compulsory General Insurance has three sources of financing: the compulsory individual contribution of members, the mandatory contribution of public or private employers and the contribution of the State. Similarly, the health services and benefits of the Rural Social Security are financed with resources from the contribution of employers, the contribution of members to the SGO, the mandatory contribution of public and private insurance, the differential contribution of families protected by the SSC, the contribution of the State and the supplementary assignments determined by the Executive Power.
\end{abstract}

Keys words: Strategy, management processes, health programs, talents, public and private insurance. 


\section{Introducción.}

La salud es un factor decisivo para el bienestar de las personas, las familias y las comunidades y, a la vez, un requisito para el desarrollo con equidad. Más aún, las personas tienen derecho a un cuidado equitativo, eficiente y atento de su salud y la sociedad en su conjunto debe garantizar que nadie quede excluido del acceso a los servicios de salud y que estos proporcionen una atención de calidad para todos los usuarios, Naciones Unidas, (2005).

Aunque por separado, las diversas actividades de salud pública tienen una larga historia. La organización de los departamentos de salud por jurisdicciones gubernamentales (ciudades, condados, distritos, naciones) empezó realmente en el siglo XIX y se centraba inicialmente en el saneamiento. Al comienzo, se prestó atención sobre todo al abastecimiento de agua potable limpia y a la eliminación inocua de desechos humanos y de otro tipo, en particular en los medios urbanos. Seguidamente, se emprendieron iniciativas para el control de las enfermedades transmisibles por otros medios, tal como la tuberculosis. A medida que se ha avanzado en el conocimiento de los factores determinantes de la salud y de las causas de enfermedad, se han ampliado los esfuerzos en materia de salud pública, Amick, et al., (1995).

Como consecuencia de la disminución progresiva observada durante el último decenio del grado de preparación, la eficacia y la accesibilidad de los recursos humanos de salud, así como la consideración en un plano secundario de este recurso vital para la salud, los países de las Américas están experimentando una crisis en sus sistemas sanitarios, que no están en condiciones de proporcionar los servicios de buena calidad que se requieren para satisfacer las necesidades sanitarias de los habitantes de la Región. Sólo con una fuerza de trabajo bien preparada, bien equipada y motivada los países podrán alcanzar los Objetivos de Desarrollo, OPS, (2007). 
Uno de los principales obstáculos que enfrentan algunas de las organizaciones que hacen parte del sector salud es la ausencia o insuficiente aplicación de métodos, procesos, y procedimientos documentados y actualizados. Es decir, falta de protocolos o criterios institucionales que garanticen procesos objetivos y ágiles. La documentación insuficiente empobrece la memoria organizacional y pasa a concentrarse en individuos que interpretan y aplican procesos a su manera, García, (2006).

Este enfoque es particularmente importante en el área de la salud pública en América Latina y el Caribe, porque en esta Región los recursos humanos y la investigación son las dos funciones de más bajo rendimiento entre las once funciones esenciales de salud pública,

"Mejorar la salud de la población" es la meta del ministerio o secretaría de salud en la mayoría de las naciones. En los últimos años, los debates de política acerca de cómo alcanzar esta meta se han ampliado para incluir el tema de las funciones esenciales de salud pública o los servicios esenciales de salud pública como los cimientos que permitirán alcanzar metas concretas. Los responsables de las políticas sanitarias han entendido que, sin una infraestructura adecuada como la que se requiere para la prestación de estos servicios, ninguna administración podrá avanzar eficientemente hacia el mejoramiento de la salud para todos. Si bien los tratamientos para enfermedades concretas y las actividades para reducir al mínimo los riesgos individuales, pueden proporcionarse con éxito uno por uno, no es posible que sean sostenibles sin una estructura de salud pública organizada. Más aun, las necesidades de una ciudad, región o nación cambiarán con el transcurso del tiempo. La presencia de una red bien desarrollada de funciones y servicios esenciales de salud pública permite la flexibilidad y el crecimiento de la programación con el correr de los años, OPS, (2005). 
Hay diversos tipos de organismos oficiales, grupos voluntarios y organizaciones privadas que participan en cierto grado en la salud pública. La mayoría de las comunidades se benefician del interés y la participación de los ciudadanos en la promoción de su propia salud mediante la educación y el cambio de comportamientos. Hasta los programas normativos tradicionales se benefician de la colaboración voluntaria de asociaciones que representan a los que deberán acatar las normas, OPS, (2005).

La Organización Panamericana de la Salud (la OPS) considera al lugar de trabajo como un entorno prioritario para la promoción de la salud en el siglo XXI. La salud en el trabajo y los ambientes de trabajo saludables se cuentan entre los bienes más preciados de personas, comunidades y países. Un ambiente de trabajo saludable es esencial, no sólo para lograr la salud de los trabajadores, sino también para hacer un aporte positivo a la productividad, la motivación laboral, el espíritu de trabajo, la satisfacción en el trabajo y la calidad de vida general. Sin embargo, pese a los beneficios comprobados que tiene, el lugar de trabajo saludable no es una realidad para gran parte de la fuerza de trabajo de América Latina y el Caribe, OPS, (2000).

La región latinoamericana enfrenta un doble desafío como consecuencia de los grandes cambios sociales económicos y demográficos; por una parte, debe enfrentar los problemas tradicionales de salud como las enfermedades contagiosas e infecciones, la mortalidad materna e infantil y por otra parte, debe lidiar con los nuevos problemas surgidos del desarrollo y de cambios paulatinos y a veces rápidos, en los perfiles de la morbilidad: el aumento de las enfermedades crónico-degenerativas, seniles y mentales y el VIH SIDA, entre otras, Arriagada, et al., (2005).

El proceso es el elemento más utilizado en los modelos de gestión de organizaciones, sobre todo en las empresas que toman la calidad total como base de su estrategia. Este interés por los 
procesos ha originado numerosas herramientas y técnicas relacionadas con la gestión de los propios procesos y con la gestión basada en los mismos. La gestión de procesos se basa en las herramientas de mejora, popularizadas por los resultados de empresas japonesas, generalmente integradas en el "método sistemático" o científico de mejora de procesos. Entre los modelos de gestión de empresa basados en los procesos se explican, por su aceptación tan amplia, el Mapa de procesos y el Cuadro de Mando Integral, Zaratiegui, (1999).

Según Regás (2009), El análisis de las implicaciones relativas a la interacción entre lo público y lo privado en relación con la salud es un ámbito extraordinariamente amplio. Antes que nada conviene reseñar que hay una vertiente ideológica en todo lo que deseamos conseguir, y al mismo tiempo en la forma como entendemos la realidad. Las formas en las que preguntamos, los métodos analíticos y la evidencia que buscamos están influidas por un sesgo ideológico. El debate sobre lo público y lo privado en las políticas de salud es vulnerable a ello, y no hay teorías asépticas al respecto.

En esta prestación interviene una gran variedad de proveedores, clínicas y aseguradoras de salud; lo que algunos autores llaman "el mercado sin organizar en salud” (Standing y Bloom, 2002). De forma adicional, se observa una amplia participación de instituciones como laboratorios, compañías farmacéuticas, escuelas para la formación de las distintas categorías de personal de salud, etc. Desde hace algunos años el sector privado ha aumentado su presencia al punto de que la colaboración con el sector público es sumamente frecuente. Este fenómeno ha sido definido como colaboración público-privada, en el campo de la salud y la importancia de contar con una sólida rectoría en el sector, que a su vez impulse una amplia intervención ciudadana en la planeación y puesta en práctica de políticas públicas, Nigenda, et al., (s/f). 


\section{Metodología.}

El presente artículo tiene lugar en este aspecto específico, la revisión de literatura más acertada sobre la investigación en la estrategia de administración en el área de salud pública-privada. Para la realización de la actual investigación, se considero, información confiable sobre trabajos plasmados, tratados en este tema, se utilizo información bibliográfica, consultas electrónicas, misma que se la obtuvo de libros electrónicos, artículos científicos y revistas.

\section{Desarrollo.}

\section{Estrategias prioritarias en la administración sanitaria}

Para Mintzberg y Quinn, (1993), una estrategia "Es el patrón o plan que integra las principales metas y políticas de una organización, y a la vez, establece la secuencia coherente de las acciones a realizar. Una estrategia adecuadamente formulada, ayuda a poner orden y a asignar, con base tanto en sus atributos como en sus deficiencias internas, los recursos de una organización, con el fin de lograr una situación viable y original, así como anticipar los posibles cambios en el entorno y las acciones imprevistas de los oponentes inteligentes,

Una estrategia es un plan de acción que se lleva a cabo para lograr un determinado fin en una empresa a largo plazo, la estrategia Empresarial Se refiere al diseño del plan de acción dentro de una empresa para el logro de sus metas y objetivos. En el campo de la administración, una estrategia, es el patrón o plan que integra las principales metas y políticas de una organización, y a la vez, establece la secuencia coherente de las acciones a realizar, Valencia, (2015). 
Constitución Política de la República del Ecuador de 2008

El texto constitucional especifica el rol y las responsabilidades del Estado en materia de salud y caracteriza a los servicios públicos de salud destacando la universalidad y la gratuidad. Si bien no se enuncia como un derecho, el acceso universal y gratuito a la salud es una consecuencia del mandato constitucional según el cual:

“[...] los servicios públicos estatales de salud serán universales y gratuitos en todos los niveles de atención y comprenderán los procedimientos de diagnóstico, tratamiento medicamentos y rehabilitación necesarios". (Art. 362). [...] el Estado será responsable [...] Universalizar la atención en salud, mejorar permanentemente la calidad y ampliar la cobertura. (Art. 363) "EI seguro universal obligatorio cubrirá las contingencias de enfermedad, maternidad, [...] El seguro universal obligatorio se extenderá a toda la población urbana y rural, con independencia de su situación laboral [...]" (Art. 369), Constitución del Ecuador, (2008).

\section{Marco constitucional y legal}

La ley Orgánica del Sistema Nacional de Salud (2002). Es la que regula la organización e institucionalidad administrativa, así como el funcionamiento del Sistema de Salud. Fundamentalmente, se basa en un esquema ya superado que necesita ser reformado. De manera general, esta legislación secundaria vigente en salud se caracteriza por cuatro defectos fundamentales que tienen que ver con su dispersión, con más de 40 leyes; repetición de normas que aparecen en más de un cuerpo normativo, con potenciales contradicciones; así como algunas leyes que, aunque formalmente vigentes, ya están en desuso o se refieren a una materia regulada por otra ley, ISAGS, (2012). 
Responsabilidades

La rectoría del Sistema Nacional de Salud está a cargo de la Autoridad Sanitaria Nacional a quien corresponde formular la Política Nacional de Salud, normar, regular y controlar todas las actividades relacionadas con la salud, así como el funcionamiento de las entidades del sector. Constituyen responsabilidades del Estado: (i) la universalización de la atención, mejorando calidad y ampliando cobertura; (ii) fortalecer los servicios estatales; (iii) garantizar las practicas ancestrales;

(iv) brindar cuidados especiales a los grupos de atención prioritarios; (v) asegurar acciones y servicios de salud sexual y reproductiva y garantizar salud integral de las mujeres, en particular durante embarazo, parto y postparto; (vi) garantizar la disponibilidad de medicamentos de calidad, seguros y eficaces y (vi) promover el desarrollo integral del personal de salud, ISAGS, (2012).

\section{Modelo político administrativo del país}

En virtud de lo que define la Constitución de la República, las competencias en salud se asignan a los distintos niveles de gobierno. Los gobiernos municipales y los distritos metropolitanos autónomos también pueden prestar directamente servicios de salud, como competencia concurrente con el nivel central. (Cuadro 1).

La particularidad en sus facultades radica en que la rectoría, planificación, regulación y control se ejercen desde el nivel central; la planificación regional, coordinación y gestión desde el nivel intermedio o regional y una fuerte coordinación y gestión desde el nivel local, Ponce, (2015). 
Cuadro 1 - Competencias en salud de los servicios de gobierno, Ecuador.

\begin{tabular}{|l|l|}
\hline Nivel de Gobierno & Competencia \\
\hline Nivel Central & Definición de políticas \\
\hline Gobiernos Municipales & $\begin{array}{l}\text { Planificación, construcción y } \\
\text { mantenimiento de infraestructura física y } \\
\text { equipamiento de salud. }\end{array}$ \\
\hline Distritos Metropolitanos Autónomos & $\begin{array}{l}\text { Planificación, construcción y } \\
\text { mantenimiento de infraestructura física y } \\
\text { equipamiento de salud. }\end{array}$ \\
\hline
\end{tabular}

Fuente: ISAGS, 2012.

Estructura territorial desconcentrada del sistema de salud

El Ministerio de Salud Pública se encuentra dentro de la denominada tipología 2 -alta desconcentración, baja descentralización-, que se caracteriza por un alto nivel de desconcentración, articulando los procesos, productos y servicios con una descentralización de baja incidencia. La particularidad en sus facultades radica en que la rectoría, planificación, regulación y control se ejercen desde el nivel central; la planificación regional, coordinación y gestión desde el nivel intermedio o regional y una fuerte coordinación y gestión desde el nivel local, ISAGS, (2012).

También se caracteriza por permitir la prestación de servicios públicos desde el nivel local a través de distritos administrativos, que son entidades territoriales comunes para los Ministerios de Salud, Educación, Inclusión Económica y Social y Trabajo, que comparten la misma tipología, lo que facilita la prestación de servicios intersectoriales. En el (gráfico 1), se muestra la diferenciación entre la estructura territorial política administrativa del país, aquella de los gobiernos autónomos y la 
estructura territorial generada por el funcionamiento eficiente de la función ejecutiva nacional en los niveles territoriales desconcentrados que contempla zonas y distritos, ISAGS, (2012).

Gráfico 1 - Estructura territorial del Sistema de Salud, Ecuador.

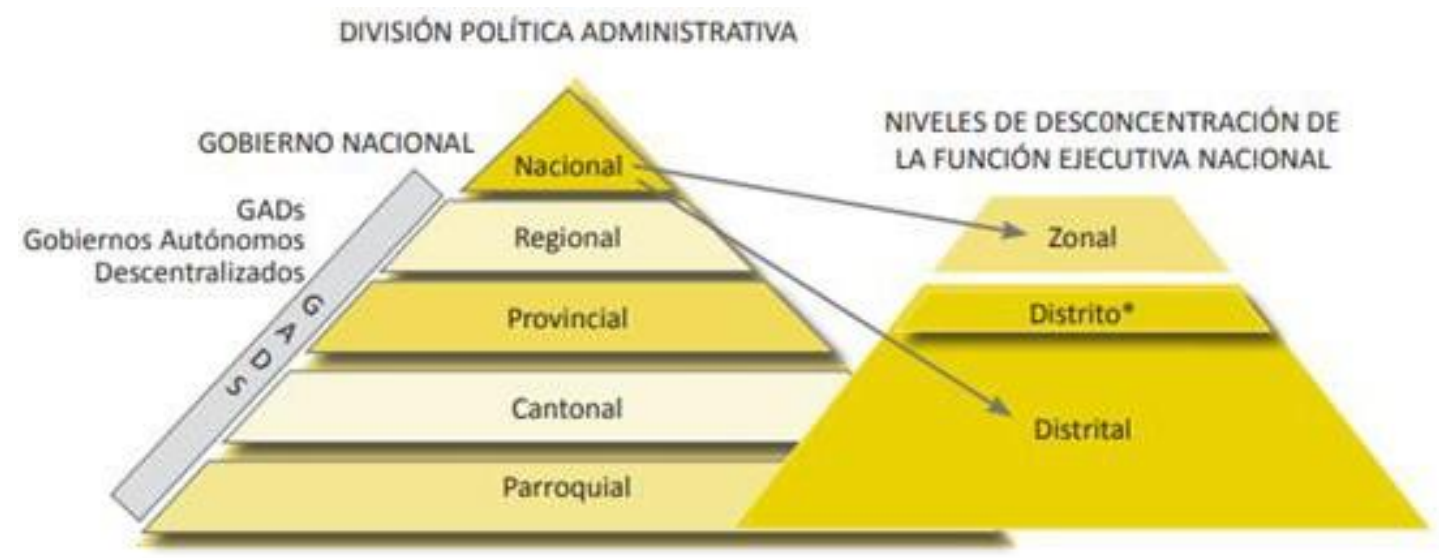

Fuente: ISAGS, 2012.

\section{Estructura y Organización}

El sistema de salud del Ecuador es mixto compuesto por dos sectores, público y privado.

El sector público comprende al Ministerio de Salud Pública (MSP), el Ministerio de Inclusión Económica y Social (MIES), los servicios de salud de las municipalidades y las instituciones de seguridad social; Instituto Ecuatoriano de Seguridad Social (IESS), Instituto de Seguridad Social de las Fuerzas Armadas (ISSFA) e Instituto de Seguridad Social de la Policía Nacional (ISSPOL)]. El MSP ofrece servicios de atención de salud a toda la población. El MIES y las municipalidades cuentan con programas y establecimientos de salud en los que también brindan atención a la población no asegurada. Lucio, et al., (2011). 
Las instituciones de seguridad social cubren a la población asalariada afiliada. El sector privado comprende entidades con fines de lucro (hospitales, clínicas, dispensarios, consultorios, farmacias y empresas de medicina prepagada) y organizaciones no lucrativas de la sociedad civil y de servicio social. Los seguros privados y empresas de medicina prepagada cubren aproximadamente a 3\% de la población perteneciente a estratos de ingresos medios y altos. Además, existen cerca de 10000 consultorios médicos particulares, en general dotados de infraestructura y tecnología elementales, ubicados en las principales ciudades y en los que la población suele hacer pagos directos de bolsillo en el momento de recibir la atención, Lucio, et al., (2011).

\section{Prestación de servicios de salud}

La Constitución del Ecuador, (2008), señala que "la salud es un derecho que garantiza el Estado, cuya realización se vincula al ejercicio de otros derechos, entre ellos el derecho al agua, alimentación, nutrición, educación, cultura física, trabajo, seguridad social, ambientes sanos y otros que sustentan el buen vivir. El Estado garantizará este derecho mediante políticas económicas, sociales, culturales, educativas y ambientales; y el acceso permanente, oportuno y sin exclusión a programas, acciones y servicios de promoción y atención integral de salud, salud sexual y salud reproductiva. La prestación de los servicios de salud se regirá por los principios de equidad, universalidad, solidaridad, interculturalidad, calidad, eficiencia, eficacia, precaución y bioética, con enfoque de género y generacional".

El Ministerio de la Salud Pública (MSP) es la Autoridad Sanitaria Nacional con capacidad de dictar normas y ejercer rectoría y control sobre el sistema. Sin embargo, es necesario que el MSP se fortalezca como ente rector del sector en la conducción, normalización, coordinación, regulación y control de las actividades de salud. El MSP también es el principal prestador de servicios en el país, 
tanto para servicios de salud colectiva como individual. Adscrito al Ministerio se encuentra el Instituto Nacional de Higiene, con responsabilidades en laboratorios de referencia, control y vigilancia sanitaria. Ponce, (2015).

El MSP, con la provisión más amplia de servicios del país, 47\% de unidades ambulatorias y hospitalarias, y los hospitales de referencia nacional más grandes del país, cubre alrededor del $51 \%$ de la población ecuatoriana, Lucio, (2010).

Los trabajadores del sector formal de la economía, incluyendo al sector campesino, tienen derecho a afiliarse al IESS. Este instituto cubre por lo menos a $20 \%$ del total de la población ecuatoriana, IESS, (2010).

Tanto en el Sistema de Seguridad Social como en el Sistema Nacional de Salud se contemplan las prestaciones de servicios de salud, así como la universalización de la atención, lo que puede generar confusiones. Sin embargo, el Sistema de Seguridad Social no tiene como objetivo la prestación de servicios de salud sino, únicamente, la de brindar un seguro que permita a sus afiliados acceder a estos servicios cuando lo requieran. Los servicios de salud, por su lado, son parte del Sistema Nacional de Salud. Ponce, (2015).

El ISSFA y el ISSPOL cubren a poco más de 5\% de la población con seguros públicos, que representan $2 \%$ de la PEA. La cobertura de salud de estos sistemas es familiar e incluye a los hijos hasta los 25 años de edad, ISSFA, (2010).

En resumen, si bien la ley proclama la cobertura universal en salud, en los hechos todavía existe una limitada cobertura real con servicios cuya calidad no siempre es la óptima. Lucio, R., (2007). 
En 1994 se promulgó la Ley de Maternidad Gratuita (LMG), que se reformó en 1998 constituyéndose en un seguro de salud que garantiza a las mujeres atención a la salud gratuita y de calidad durante su embarazo, parto y posparto, así como acceso a programas de salud sexual y reproductiva, LMG, (1994).

Esta ley también ampara la atención a la salud a los recién nacidos y los menores de 5 años como una acción de salud pública gratuita responsabilidad del Estado. En 2007 el MSP asumió la LMG como un programa regular, antes de ser un programa regular del MSP, cubría por separado los gastos en medicinas, insumos, micronutrientes, suministros, exámenes básicos de laboratorio y exámenes complementarios para la atención de las mujeres embarazadas, los recién nacidos y los menores de 5 años de edad.18 En la actualidad complementa las prestaciones regulares del MSP, Hermida, et al., (2005).

Los ecuatorianos que mayores beneficios en salud reciben son aquellos que están cubiertos por alguno de los institutos de seguridad social. Los beneficiarios del IESS están protegidos contra la contingencia de enfermedad por las siguientes prestaciones: asistencia médica, quirúrgica, dental y farmacológica integral. Estas prestaciones incluyen consulta externa, urgencias, medicina curativa, preventiva y de rehabilitación mediante la provisión de ortesis y prótesis; atención quirúrgica y hospitalización; atención médica domiciliaria; subsidio en dinero por enfermedad, y atención en unidades médicas ajenas al IESS. Los beneficiarios del IESS también reciben compensación de gastos médicos y atención médica mediante convenios suscritos con diversas clínicas privadas, IESS, (s/f).

El ISSFA ofrece a sus miembros servicios de consulta externa, emergencia, hospitalización y medicina ambulatoria para pacientes aquejados por padecimientos crónicos graves. El titular tiene 
una cobertura de $100 \%$, mientras que sus dependientes, excluyendo a los padres, tienen cobertura sólo para ciertas enfermedades. El ISSPOL otorga servicios de asistencia médica, quirúrgica, obstétrica, odontológica, de hospitalización y farmacéutica, prótesis, ortesis y rehabilitación, así como acciones de medicina preventiva, Ley de Seguridad Social de las Fuerzas Armadas, (1992), Ley de Seguridad Social de la Policía Nacional, (1995) y Superintendencia de Bancos y Seguros del Ecuador, (s/f).

Se destaca el crecimiento importantísimo del sector privado con fines de lucro que alcanzó el 27,03\% de los egresos nacionales en 2008, además se resalta fácilmente las actividades hospitalarias como un nicho del sector privado. Modelo de Atención Integral de Salud con Enfoque Familiar, Comunitario e Intercultural. Tras la adopción de la política de gratuidad, en el año 2007 se crea la Subsecretaria de Extensión y Protección Social en Salud y el Programa de Aseguramiento Universal de Salud se transforma en el Modelo de Atención Integral de Salud con Enfoque Familiar, Comunitario e Intercultural (MAIS). Ponce, (2015).

El modelo propuesto por el MSP busca consolidar la atención primaria de la salud como estrategia que prioriza la prevención de la enfermedad, la promoción de la salud; priorización desde un enfoque de evitabilidad y la participación organizada de los sujetos sociales en la construcción de una sociedad saludable. En este marco se contrataron 4.582 profesionales que integrarían los Equipos Básicos de Salud (EBAS), instrumento estratégico del modelo. Con ello se incrementó la oferta de servicios y la cobertura de la red del Ministerio de Salud Pública. Ponce, (2015).

Primer nivel de atención. Es el más cercano a la población, facilita y coordina el flujo del paciente dentro del sistema, garantiza una referencia y contra referencia adecuada y asegura la continuidad y longitudinalidad de la atención. Promueve acciones de salud pública de acuerdo a la 
norma emitida por la Autoridad Sanitaria. Es ambulatorio y resuelve problemas de salud de corta estancia; además, se constituye en la puerta de entrada obligatoria al Sistema Nacional de Salud. Dentro de este nivel se encuentran los siguientes tipos de establecimientos: • Puesto de salud, • Consultorio general, • Centro de salud rural, • Centro de salud urbano, • Centro de salud urbano de 12 horas, $\bullet$ Centro de salud urbano de 24 horas. ISAGS, (2012).

Segundo nivel de atención. Comprende todas las acciones y servicios de atención ambulatoria especializada y aquellas que requieren hospitalización; constituye el escalón de referencia inmediata del I nivel de atención. Se desarrollan nuevas modalidades de atención no basadas exclusivamente en la cama hospitalaria, tales como cirugía ambulatoria y hospital del día. Comprende los siguientes tipos de establecimientos:

- Consultorio de especialidades clínico quirúrgicas, • Centro de especialidades, • Centro clínico quirúrgico ambulatorio (hospital del día), • Hospital básico, • Hospital general.

Tanto las unidades de primero como de segundo nivel tienen un área geográfica delimitada y una población definida de cobertura. En todos los establecimientos de salud se aplicará la estrategia de atención primaria de salud acorde a las necesidades de la población. ISAGS, (2012).

Tercer nivel de atención. Corresponde a los establecimientos que prestan servicios ambulatorios y hospitalarios de especialidad y especializados. Los centros hospitalarios son de referencia nacional; resuelven los problemas de salud de alta complejidad, tienen recursos de tecnología de punta, intervención quirúrgica de alta severidad, realizan trasplantes, cuidados intensivos, cuentan con subespecialidades reconocidas por ley y se incluyen los siguientes: • Centro especializado, • Hospital especializado, • Hospital de especialidades. ISAGS, (2012). 
Enfermedades catastróficas o cuarto nivel de atención. Como política de estado está la atención de las enfermedades consideradas como catastróficas, que para su tratamiento tienen financiamiento del Estado para permitir la gratuidad de su atención. Este programa, denominado Programa de Protección Social en Salud (PPS), está manejado por el Ministerio de Inclusión Económica y Social e incluye hasta el momento, sin descartar su progresividad: malformaciones congénitas del corazón, cáncer, tumores cerebrales, insuficiencia renal crónica, trasplantes de riñón, hígado, médula ósea, malformaciones vasculares, secuelas de quemaduras, prótesis externas, implantes cocleares y órtesis. Los servicios prestados por los Programas Especiales de Protección Social en Salud (PPS) se han incrementado, generando al año 2010 un ahorro de bolsillo en la población que asciende a los USD 25 millones. Estos han cubierto sobre todo atención a problemas oculares, diabetes, implantes y cardiopatías, entre otros. ISAGS, (2012).

\section{La prevención de enfermedades}

Según Ponce, (2015). En el ámbito de prevención, especialmente en lo relacionado con las enfermedades transmisibles en el país, el MSP está llevando adelante una serie de programas de salud pública denominados "programas verticales", que incluyen acciones de prevención (y curación) en materia de tuberculosis, HIV - SIDA y el programa Servicio Nacional de Erradicación de la Malaria para enfermedades transmisibles por vectores, cuyos logros se detallan a continuación.

El Programa Ampliado de Inmunizaciones del Ecuador (PAI), es uno de los programas más exitosos de América Latina y ha recibido varios reconocimientos de la Organización Mundial de la Salud y otros organismos internacionales. El PAI mantiene al país desde hace 20 años sin poliomielitis, 14 años sin sarampión, 10 años sin fiebre amarilla, 6 años sin rubeola y ha eliminado el tétanos neonatal como problema de salud pública, entre otros logros importantes; a pesar de los 
rebrotes de estas enfermedades en varios países del mundo. La malaria en el Ecuador ha tenido un franco descenso, mucho más pronunciado en los años 2010 y los primeros meses del 2011. Ponce, (2015).

La oncocercosis, enfermedad que causaba graves daños en la población del norte de Esmeraldas, incluyendo muchos casos de ceguera, hoy se encuentra en etapa de precertificación de su eliminación y se aspira a que para el año 2013 se certifique definitivamente la eliminación. El Ministerio de Salud Pública actualmente garantiza el tratamiento al $100 \%$ de los pacientes diagnosticados y registrados de SIDA. En el ámbito de la nutrición, Ecuador, al igual que la mayoría de los países, presenta simultáneamente dificultades de déficit nutricional y de exceso y desbalance. Ponce, (2015).

\section{La vigilancia}

La Subsecretaría de Vigilancia de la Salud Pública del MSP tiene por misión analizar, vigilar y velar por la situación de salud pública de la población mediante el diseño de políticas, regulaciones, planes y proyectos en los ámbitos de vigilancia epidemiológica, vigilancia y control sanitario y por estrategias de salud colectiva. La mencionada Subsecretaría está compuesta por:

La Dirección de Vigilancia Epidemiológica, La Dirección de Control Sanitario y El nuevo modelo de atención del MSP incorporará experiencias innovadoras y eficaces, como la epidemiología comunitaria. Ponce, (2015).

El fortalecimiento de la vigilancia epidemiológica y del sistema de información en salud, a fin de detectar de manera temprana y oportuna los brotes epidémicos y las enfermedades crónicas no transmisibles evitando que éstas se transformen en enfermedades catastróficas; la consolidación de 
las estrategias de prevención y control -las cuales requieren fortalecerse en el marco del proceso de reforma-; la implementación del MAIS y su materialización en rutinas de atención para los enfermos agudos pero sobre todo para los portadores de enfermedades crónicas no transmisibles, a fin de asegurar la continuidad de los cuidados; y la construcción efectiva de redes integradas de provisión de servicios de salud con mecanismos estables y eficientes de referencia y contrarreferencia. Espinosa, et al., (2017).

\section{Sistema de financiamiento de salud}

La Constitución en el artículo 366 estipula lo siguiente: "El financiamiento público en salud será oportuno, regular y suficiente, y deberá provenir de fuentes permanentes del Presupuesto General del Estado. Los recursos públicos serán distribuidos con base en criterios de población y en las necesidades de salud", Constitución del Ecuador, (2008).

Según la Ley de Seguridad Social 2001, el Seguro General Obligatorio (SGO) del IESS tiene tres fuentes de financiamiento: la aportación individual obligatoria de los afiliados, la aportación obligatoria de los empleadores públicos o privados y la contribución del Estado. Señala también que los servicios de salud y prestaciones del Seguro Social Campesino (SSC) se financian con recursos provenientes de las siguientes fuentes: el aporte solidario de los empleadores, el aporte de los afiliados al SGO, la contribución obligatoria de seguros públicos y privados, el aporte diferenciado de familias protegidas por el SSC, la contribución del Estado y las asignaciones suplementarias determinadas por el Poder Ejecutivo, Ley 55, (2001).

El ISSPOL se financia con un aporte de los miembros de la policía en servicio activo de $2.5 \%$ de su salario y un aporte del patrono (Estado) de 3 por ciento. Es el único caso en el país 
donde también los retirados aportan 2.5\% de sus pensiones, Seguridad Social de la Policía Nacional, (1995).

En el caso del ISSFA, los recursos provienen de los aportes de los militares en servicio activo (equivalente al 3.35\% de su haber militar) y los aportes patronales que hace el Ministerio de Defensa $(5.85 \%$ del haber militar del personal en servicio activo y $0.52 \%$ del $2 \%$ del haber militar de un soldado en servicio activo, multiplicado por el número de aspirantes a oficiales, tropa y conscriptos), Ley de Seguridad Social de las Fuerzas Armadas, (1992) y Superintendencia de Bancos y Seguros del Ecuador, (s/f).

El sistema de financiamiento de la salud vigente en el Ecuador es un sistema mixto conformado por tres subsistemas principales:

- Subsistema de base tributaria, para los servicios de salud que presta el Gobierno, principalmente mediante las entidades prestadoras del Ministerio de la Salud Pública (MSP).

- Sistema público de Seguro Social de Salud, orientado al sector formal, sea general (IESS) o especifico (ISSFA, ISSPOL, SSC) y financiado mayormente mediante contribuciones.

- Subsistema privado, con seguros privados y pagos de bolsillo. 
Gráfico 2- Modelo de financiamiento del sistema de salud de Ecuador

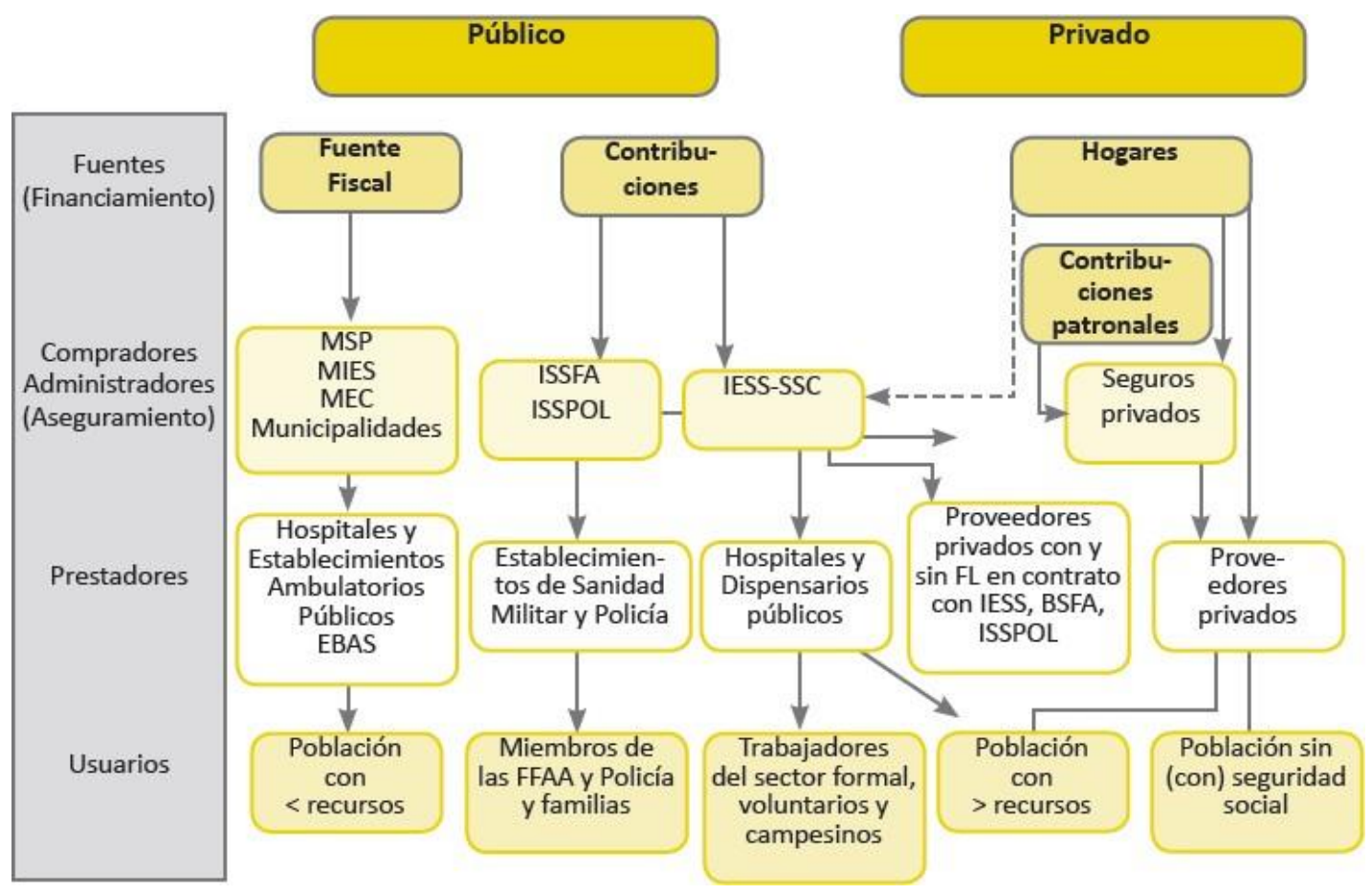

Fuente: MCDS, 2010: Hacia la seguridad social universal contingencia de enfermedad, maternidad.

Como se puede observar, el financiamiento de las entidades públicas del gobierno (MSP;

MIES, ME; gobiernos locales) proviene de fuentes fiscales, en el caso de las entidades públicas, del Sistema Nacional de Seguridad Social. La financiación se realiza a través de cotizaciones pagadas por los empleadores y trabajadores. Mientras tanto, los seguros privados y otros esquemas prepagos solo son accesibles a los estratos de altos ingresos. Los hogares de ingresos bajos también participan en el financiamiento del sector privado en salud a través de pagos directos al punto de servicios cuando sus demandas no han sido satisfechas por el sector público. ISAGS, (2012). 
Infraestructura y equipos para la prestación de servicios de salud

Ecuador tiene registrados 3847 establecimientos de atención a la salud, de los cuales $80 \%$ pertenecen al sector público. El MSP concentra 47\% de las unidades y el IESS otro 24 por ciento. Una pequeña proporción (10\%) pertenece a municipios, ministerios diferentes al MSP, ISSFA, ISSPOL y otras entidades. El sector privado concentra alrededor del $20 \%$ de los establecimientos de salud, $14 \%$ en instituciones con fines de lucro y el restante $6 \%$ en organismos que ofrecen atención a la salud sin fines de lucro, INEC, (2007).

La mayor parte de los establecimientos con internación registrados en 2007 (545) eran clínicas particulares, que representan $75 \%$ del total. Le siguen en importancia los hospitales cantonales, los hospitales generales y, en una mínima proporción, los hospitales de especialidad. La mayor parte de los establecimientos sin internación que se ubican en las áreas urbanas son dispensarios médicos. En las zonas rurales, la mayor proporción corresponde a subcentros de salud, a los que siguen los dispensarios médicos, INEC, (2007).

El MSP cuenta con 1674 establecimientos de salud ambulatorios y 125 hospitales: 28 hospitales generales, 79 hospitales cantonales y 18 hospitales de especialidad. El IESS brinda atención en 902 unidades ambulatorias entre servicios y anexos en las empresas, además de 18 centros hospitalarios, tres regionales y 15 provinciales. El ISSFA dispone de 72 establecimientos con servicios de atención ambulatoria y 16 de hospitalización. El ISSPOL cuenta con 35 unidades operativas, de las cuales dos son hospitalarias, INEC, (2007).

Por parte del MSP, el incremento del número de establecimientos de salud es relevante en los últimos años. La tasa de crecimiento ha revertido la tendencia registrada durante los últimos 15 años, lo que evidenciaba una retirada del Estado en ámbito de salud. Solo durante el año 2010 se 
crearon 132 unidades de salud, 497 se remodelaron, 362 unidades disponen de monitoreo y realizan visitas promocionales de salud, 13 hospitales fueron ampliados, remodelados y equipados. MCDS, (2011).

El sistema de salud ecuatoriano sin duda se ha fortalecido con el profundo y ambicioso proceso de reforma, el cual ha sido posible gracias a un apoyo político explícito y sostenido, expresado en la priorización de la salud como un derecho de todos y de todas. No obstante, existen grandes retos aún pendientes, entre los cuales se destacan la implementación de un modelo de financiamiento sostenible con un fondo mancomunado para el sistema público, que permita una mayor eficiencia en el gasto en salud y garantice la sostenibilidad del sistema en el mediano plazo, Espinosa, et al., (2017).

Participación del sector privado en la implementación del modelo de atención

La participación del sector privado en la implementación del modelo de atención se operativiza mediante la estructuración de la "red complementaria": servicios que el sector público compra al sector privado para atender a la demanda que no puede ser cubierta por este sector y que funciona mediante convenios de gestión suscritos entre las instituciones de salud del sector público y del sector privado. La Red se encuentra en la fase de estructuración, lo que implica regular los servicios que se prestan a través de la red complementaria, emitir las acreditaciones a prestadores institucionales, la normativa que permita el funcionamiento de estas entidades privadas y exigir el registro nacional, zonal y distrital de los profesionales de la salud con sus especialidades médicas y odontológicas. ISAGS, (2012).

El nuevo marco legal y político del modelo de sistema de salud del Ecuador. Ha logrado romper con el antagonismo entre eficiencia económica e inversiones en el sector social, reafirmado 
el rol del Estado, la soberanía y autodeterminación en la formulación participativa de políticas públicas y programas incluyentes y movilizadores por la salud y la vida desde lo local, pero además garantiza de manera progresiva el acceso de toda la población a servicios de salud e infraestructura sanitaria, erradicando las inequidades, la exclusión y la discriminación. Se recupera la salud como un derecho humano que participa de la construcción del bienestar como un hecho colectivo. ISAGS, (2012).

Macrogestión

Rectoría

El Ministerio de Salud Pública MSP es la Autoridad Sanitaria Nacional ASN, núcleo de pensamiento estratégico del sistema nacional de salud, conductor de la red pública de servicios; que planifica, regula, genera política pública; define estándares y mecanismos de gestión articulación; con el objetivo de vigilar la salud pública y actuar a través de la provisión de servicios de promoción, prevención, curativos y de rehabilitación, así como actuar, a través del control de todos los productos y actividades que afectan a la salud, hacia mejoras del nivel de salud de la población ecuatoriana. ISAGS, (2012).

El Ministerio de Salud Pública ejercerá plenamente la gobernanza del Sistema Nacional de Salud con un modelo referencial en Latinoamérica que priorice la promoción de la salud y la prevención de enfermedades, con altos niveles de atención de calidad, con calidez, garantizando la salud integral de la población y el acceso universal a una red de servicios, con la participación coordinada de organizaciones públicas, privadas y de la comunidad. ISAGS, (2012). 
Soluciones estructurales para el fortalecimiento de la autoridad sanitaria nacional

La nueva estructura orgánica del Ministerio de Salud Pública propone la creación de tres entidades especializadas: la Coordinación General de Desarrollo Estratégico en Salud, la Subsecretaría de Gobernanza de la Salud y la Agencia de Control para los establecimientos de Salud, adscrita al Ministerio. Gráfico 3. ISAGS, (2012).

Gráfico 3 - Soluciones estructurales para el ejercicio del rol de Autoridad Sanitaria Nacional

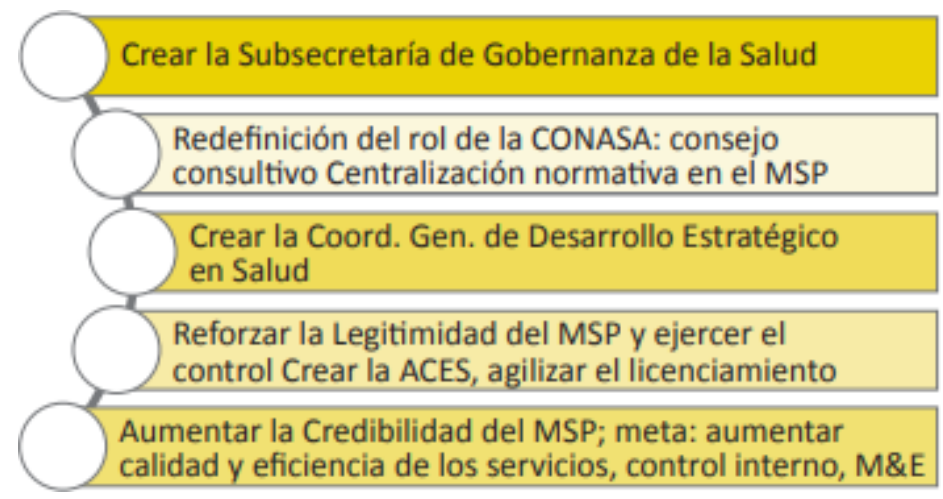

Fuente: Ministerio de salud pública del Ecuador.

Formulación de políticas y planes

El Ecuador cuenta con una Política Nacional de Salud que fue aprobada luego de un proceso participativo de consulta nacional a los diferentes actores del Estado y la Sociedad Civil, promovida por el Ministerio de Salud Pública y el Conasa en el periodo 2002-2006.

La política, aunque poco difundida, se encuentra vigente y está organizada en tres ámbitos de acción: la construcción de ciudadanía en salud, la protección integral de salud y el desarrollo sectorial. Sus objetivos son: 
- Promover la ciudadanía en salud, la garantía, el respeto, la promoción, la protección y la exigibilidad de los derechos humanos para el ejercicio de una vida digna y saludable.

- Garantizar la protección integral de la salud de la población ecuatoriana facilitando los medios para promover la salud, tanto física como mental, prevenir y enfrentar las enfermedades y sus causas, mitigando sus efectos biológicos, económicos y sociales.

- Desarrollar las capacidades del sector de la salud mediante procesos organizativos y participativos que conduzcan al establecimiento y funcionamiento del Sistema Nacional de Salud, el desarrollo integral del talento humano, el desarrollo científico y tecnológico y el impulso a los sistemas de información y la dotación de recursos materiales, tecnológicos y financieros.

Modelo de gestión

Se definió un nuevo modelo de gestión y estructura organizacional cuyos objetivos buscaban corregir las ineficiencias evidenciadas en la institución; modernizar la administración y el manejo gerencial interno para una mayor efectividad, eficacia y transparencia, fortaleciendo su capacidad para ejercer el rol de autoridad sanitaria, y llevar a cabo estrategias y programas en vista del cumplimiento de su misión, objetivos institucionales y mandatos a su cargo. Se plantea la creación de la Subsecretaria de Prevención y Promoción de la Salud con el objetivo de sustentar el modelo de atención primaria que tiene un enfoque hacia la promoción, prevención y reestructuración de redes. Ponce, (2015).

Vigilancia en Salud

Implementación del Reglamento Sanitario Internacional 
El Reglamento Sanitario Internacional (RSI) tiene el propósito de: "prevenir la propagación internacional de enfermedades, proteger contra esa propagación, controlarla y darle una respuesta de salud pública proporcionada y restringida a los riesgos para la salud pública y evitando al mismo tiempo las interferencias innecesarias con el tráfico y el comercio internacionales". Ponce, (2015).

La red de laboratorios para la vigilancia de la Salud Pública está constituida por el Instituto Nacional de Higiene Leopoldo Izquieta Pérez, establecido en la ciudad de Guayaquil. Las 21 provincias tienen un laboratorio para la vigilancia en salud que son de menor complejidad que los zonales. En casos específicos participan laboratorios externos al MSP con el objeto de mejorar el diagnóstico laboratorial. Ponce, (2015).

\section{Vigilancia epidemiológica}

Como parte del Sistema Integrado de Vigilancia Epidemiológica está el SIVE ALERTA, que permite la notificación inmediata, la investigación e intervención correspondiente (tiene un software específico como herramienta de apoyo). Está implementado en todas las unidades operativas de las 24 provincias, en las 170 Áreas de Salud y en los Hospitales Provinciales especializados, generales y básicos. El país no dispone de un sistema de vigilancia de los principales factores de riesgo para enfermedades crónicas no trasmisibles, tabaco, consumo excesivo de alcohol, sedentarismo y alimentos no saludables. Ponce, (2015).

\section{Vigilancia sanitaria}

Una de las acciones de salud pública que debe ejecutar la Autoridad Sanitaria Nacional es la vigilancia y control sanitario de establecimientos y productos de uso y consumo humano. La Dirección de Control y Mejoramiento en Vigilancia Sanitaria, perteneciente al Ministerio de Salud 
Pública, realiza funciones que abarcan la regulación y normatización técnica para registro, vigilancia y control de establecimientos y productos de uso y consumo humano. Ponce, (2015).

\section{Vigilancia ambiental en salud}

Salud Ambiental tiene como función la vigilancia de los impactos ambientales en la salud de la comunidad. Sus componentes son: la vigilancia de la calidad del agua de consumo humano; el manejo adecuado de los plaguicidas; vigila las intoxicaciones por plaguicidas; salud y seguridad ocupacional, que incorpora al servicio de salud acciones que permiten evaluar las condiciones de trabajo, especialmente en los procesos peligrosos, accidentes laborales, promoción y prevención adecuadas. Ponce, (2015).

Ecuador cuenta con la Secretaría de Riesgos, que tiene una Dirección de Gestión de Riesgos en cada uno de los ministerios del sector público. Estas últimas son responsables de llevar adelante la preparación de planes de contingencia en casos de desastre. El MSP cuenta con esta Dirección que coordina con epidemiología cuando se trata de eventos de salud pública de interés nacional o internacional. Ponce, (2015).

ARCSA, (s/f), Los objetivos estratégicos principales del nuevo modelo de gestión de la Agencia Nacional de Regulación, Control y Vigilancia Sanitaria (ARCSA) son los siguientes:

- Incrementar la eficacia en la regulación de productos de uso y consumo humano y establecimientos bajo su ámbito de competencia contribuyendo a la salud pública.

- Incrementar la eficacia y eficiencia en la certificación, vigilancia y control posterior de productos y establecimientos bajo su ámbito de competencia contribuyendo a la mejora de la salud de la población. 
- Incrementar el desarrollo de Talento Humano de ARCSA.

- Incrementar la Eficiencia Operacional de ARCSA.

- Incrementar el uso eficiente de presupuesto de ARCSA.

\section{Recursos humanos que prestan los servicios}

Aunque entre 1996 y 2010 se incrementó la cantidad de recursos humanos empleados dentro del sistema de salud en aproximadamente $21 \%$, en Ecuador todavía prevalece un déficit relativo de médicos y enfermeras, que se agrava por un sistema de contratación laboral en el sector público de cuatro y seis horas. En 2007 Ecuador contaba con alrededor de 75 mil trabajadores de la salud. La razón de médicos por 1000 habitantes era de 1.9, muy inferior a los países como Argentina (3.0) y Uruguay (3.6). La tasa de enfermeras por 1000 habitantes a nivel nacional era de 0.6, que está por debajo del promedio latinoamericano, que en 2000 era de 0.8. INEC, (2007).

El grueso de los médicos y enfermeras trabajan en el MSP y no se tiene información precisa sobre el número de médicos y enfermeras que trabajan en el sector privado. La tendencia institucional de contratación de recursos humanos para 2010 parecer haber cambiado, ya que el IESS se encuentra realizando contrataciones de manera importante para cubrir el incremento de cobertura aprobado. Los médicos tienden a concentrarse en las grandes ciudades, lo que genera problemas serios de distribución. En la provincia de Pichincha hay 2 médicos por 1000 habitantes, mientras que en las provincias de Galápagos y Orellana esta razón es de apenas 0.56 y 0.43 , respectivamente. INEC, (2007).

Es necesario desarrollar una propuesta conjunta (Universidad - Ministerio) del profesional del área de la salud que requiere el Sistema Nacional de Salud y los nuevos retos que se plantean en los ámbitos del desarrollo humano, familiar, social y laboral, como consecuencia de las 
transformaciones científicas, tecnológicas, económicas, comerciales y políticas que caracterizan a la sociedad actual, en cumplimiento de la Constitución y las leyes. Ponce, (2015).

El Ecuador cuenta con 75 universidades, 145 extensiones y 285 institutos. Entre las universidades, 11 tienen clasificación "A" y cumplen con los requisitos completos de acreditación y ofrecen carreras de formación de pregrado y posgrado en medicina y carreras afines a la salud. Existen también otras universidades con calificación B y C. CONEA, (2011).

La cantidad y calidad de la oferta de la fuerza de trabajo aún no es suficiente para atender las necesidades del sistema de salud en el país. Según E. Granda, existía una ruptura del contrato social, de inseguridad y conflictividad laboral en salud, de baja calidad y productividad de servicios, de precariedad del trabajo y de desocupación creciente. El número de profesionales se ha incrementado entre los años 2000 y 2009, como se puede observar en la Tabla 1. Podríamos señalar que esto está de acuerdo con el crecimiento poblacional y de universidades formadoras en el país.

Tabla 1 - Número de personal que trabaja en el sector salud, Ecuador, 2000-2009

\begin{tabular}{|c|c|c|c|c|c|c|}
\hline \multirow{2}{*}{ Profesionales } & \multicolumn{2}{|c|}{ Sector público } & \multirow{2}{*}{$\begin{array}{c}\text { Crecimiento } \\
\%\end{array}$} & \multicolumn{2}{|c|}{ Sector privado } & \multirow{2}{*}{$\begin{array}{c}\begin{array}{c}\text { Crecimiento } \\
\%\end{array} \\
\text { Sector privado }\end{array}$} \\
\hline & 2000 & 2009 & & 2000 & 2009 & \\
\hline Médicos & 9.733 & 15.048 & 55 & 8.602 & 14.223 & 65 \\
\hline Enfermeras & 5.326 & 8.934 & 68 & 994 & 1.823 & 83 \\
\hline Odontólogos & 1.913 & 3.073 & 61 & 149 & 290 & 95 \\
\hline Obstetrices & 844 & 1.310 & 55 & 193 & 246 & 27 \\
\hline $\begin{array}{l}\text { Bioquímicos- } \\
\text { Farmacéuticos }\end{array}$ & 283 & 415 & 47 & 204 & 249 & 22 \\
\hline $\begin{array}{l}\text { Licenciados y/o } \\
\text { tecnólogos }\end{array}$ & 2.156 & 3.191 & 48 & 779 & 1.389 & 78 \\
\hline $\begin{array}{l}\text { Auxiliares de } \\
\text { Enfermeria }\end{array}$ & 10.902 & 12.035 & 10 & 2.327 & 3.416 & 47 \\
\hline $\begin{array}{l}\text { Personal de } \\
\text { Servicios }\end{array}$ & 7.968 & 9.119 & 14 & 2.103 & 3.070 & 46 \\
\hline
\end{tabular}

Fuente: Anuario de Recursos y Actividades de Salud-INEC, 2009. 
En el año 2014, de acuerdo al Observatorio de Recursos Humanos de OPS, Ecuador presentaba la siguiente densidad de profesionales de salud por 10.000 habitantes.

Tabla 2 - Número de profesionales de salud por 10.000habitantes en Ecuador, 2014.

\begin{tabular}{ccc} 
Médicos & Enfermeros & Dentistas \\
\hline 20.4 & 10.1 & 2.8 \\
\hline
\end{tabular}

Fuente: Plataforma de asimetrías en salud SurM.A.S. Disponible en: http://isags-unasur.org/dados/

La distribución geográfica, tanto aquella que se refiere a universidades como a la concentración de profesionales de la salud, es bastante desigual entre las diversas zonas del país.

Los médicos están concentrados en los hospitales y grandes centros urbanos. El informe de consultoría realizado por el Consejo Nacional de Salud (CONASA) en el año 2008, que analiza cada uno de los tipos de recursos humanos que labora en el sector salud, indica que las clínicas y los hospitales generales agrupan los mayores porcentajes de médicos $(63,0 \%)$. En cambio, los servicios de atención ambulatoria que brindan atención primaria solo tienen una cuarta parte $(24,9 \%)$ del total de médicos. CONASA, (2008).

. En el caso de los odontólogos, son los Subcentros de Salud, seguidos por los dispensarios médicos, los que presentan porcentajes más elevados. Las enfermeras se ubican mayoritariamente en los hospitales generales $(43,4 \%)$ y en los Subcentros de Salud. Las Obstetrices se encuentran principalmente en los Subcentros $(39,0 \%)$ y luego en los Centros de Salud. Por su parte, el 44,2\% de los tecnólogos médicos se encuentran en hospitales generales y el 20,9\% en las clínicas particulares. CONASA, (2008). 
Estrategias de desarrollo laboral para los profesionales de la salud

Las funciones de salud pública abarcan gran parte de la fuerza laboral de salud en general y, para llevar a cabo adecuadamente las actividades en este campo, los trabajadores de salud deben realizarlas junto con las responsabilidades primarias de su cargo, en particular aquellos que prestar servicios de primera línea a nivel de atención primaria. Además, los trabajadores que llevan a cabo actividades de salud pública no se limitan al sector sanitario, sino que incluyen los llamados trabajadores de salud pública indirectos o "secundarios", Rosenberg y Lovell, (2006).

Muchos países de la Región de las Américas y extrarregionales, con la finalidad de fortalecer su infraestructura sanitaria y, en consecuencia, desarrollar los recursos humanos del sector, han comenzado a planificar su desarrollo tratando de definir su "fuerza de trabajo" de salud pública. Los países han adoptado distintos enfoques para superar el reto de definir esta compleja fuerza de trabajo de una manera que incluya tanto a las instituciones y entidades como a las personas que participan en las actividades necesarias para el desempeño de las funciones esenciales de salud pública, independientemente de que esa labor conste de forma específica en la descripción del puesto o entre sus responsabilidades, OPS, (2007).

Otro aspecto fundamental de la reforma es la gran inversión realizada en recursos humanos y el desarrollo de diversas estrategias destinadas a cubrir la brecha de profesionales de la salud, entre las cuales se encuentran el programa "Ecuador saludable por ti vuelvo", orientado a promover el retorno de profesionales de la salud ecuatorianos para cubrir el déficit a corto plazo; la creación de la carrera de Técnico de Atención Primaria de Salud y el financiamiento de más de 1500 becas para su formación; el reconocimiento del médico familiar como especialista, con un incremento acorde de su salario; la entrega de más de 3600 becas de especialización; la creación del puesto de gerente de 
hospital para los hospitales de más de 70 camas; y la contratación de más de 5000 profesionales de la salud entre 2012 y 2015, Espinosa, et al., (2017).

Como resultado de la implementación de estas estrategias, en el periodo 2008-2015 se triplicó el número de profesionales de la salud contratado por el MSP, pasando de 11201 a 33 644, y se cuadruplicó su salario, el cual se ubicaba en un rango de US\$ 919 a US\$ 1197 mensuales y se elevó a un rango de US\$ 1676 a US\$ 4000 mensuales con bono de residencia, Espinosa, et al., (2017).

La problemática laboral de los profesionales de la salud había sido abandonada históricamente por el ente rector sin brindar soluciones visibles a sus demandas. Como bien se menciona en el Informe sobre la Salud en el Mundo de la OMS de 2006, es necesario que el recurso humano reciba una remuneración ajustada a su trabajo y esfuerzo, pues este afecta la calidad del servicio que otorga. Por este motivo, la Autoridad Sanitaria, enfocada a brindar soluciones en el desarrollo laboral del personal de sanitario, identificó la necesidad de disponer de incentivos tanto económicos como de orden general, que motiven al personal, ya que se ha comprobado que los incentivos suelen ser más eficaces cuando incluyen un grupo de beneficios, Espinosa, et al., (2017).

Antes de la reforma, los profesionales sanitarios del subsistema público en Ecuador contaban con una remuneración que no era competitiva con el salario ni con los incentivos que se ofrecían en el sector privado. En esta área, el MSP realizó un esfuerzo importante para mejorar la situación laboral del personal sanitario. En relación con los médicos, su salario aumentó aproximadamente $80 \%$ cuando se trabajó en una homologación de salarios en el sector público y se estipularon incentivos a zonas de difícil acceso para prevenir la migración de profesionales, Malo y Malo (1014), LOSEP, (2010) y Ministerio de Relaciones Laborales, (2012). 
El aumento de salarios de los profesionales de la salud fue uno de los objetivos iniciales que tuvo mayor resonancia, puesto que no benefició únicamente a los médicos, sino también a enfermeras y profesionales de la asistencia sanitaria. Como se sostiene firmemente que el personal sanitario es un pilar para reforzar los sistemas de salud y que se debe invertir en ellos, Espinosa, et al., (2017).

Con una mejor remuneración se pudo dar inicio a un trabajo complejo relativo al diseño e implantación de incentivos adicionales para los profesionales sanitarios. La remuneración homologada cumplió con una finalidad adicional al aumento salarial: evitar la migración de los profesionales dentro del país hacia las ciudades más grandes, Malo y Malo (1014).

El MSP garantiza la disponibilidad de profesionales de calidad en todo el territorio nacional, aportando así recursos al correcto funcionamiento del Modelo de Atención Integral de Salud (MAIS). Junto con un salario homologado, se identificó que existían áreas de difícil acceso en las cuales se necesitaba trabajar con el Ministerio del Trabajo (MDT), para motivar a los profesionales de la salud a ofrecer sus servicios en ellas. Por tal motivo surgió la Norma para bonificación geográfica a servidores del sector salud, Ministerio de Relaciones Laborales, (2012).

Para cubrir la necesidad de profesionales sanitarios preparados es importante prever adicionalmente su etapa de inserción al sistema laboral. Se debe asegurar un entorno laboral favorable, OPS, (2008).

El MSP formuló una normativa de apoyo y mejora de la situación de estudios y laboral del Interno Rotativo. El Internado Rotativo en Ecuador tiene un salario que previamente se negociaba aleatoriamente en ausencia de normas. El MSP, junto con el MDT, elaboró la Norma Conjunta de Internado Rotativo, MDT y MSP, (2015). 
Esta Norma menciona claramente el horario del interno, su salario y se convierte en obligatoria su afiliación al Instituto Ecuatoriano de Seguridad Social (IESS), un beneficio laboral adicional para su correcto desempeño, Espinosa, et al. (2017).

\section{Conclusiones.}

Una estrategia adecuadamente formulada, ayuda a poner orden y a asignar, con base tanto en sus atributos como en sus deficiencias internas, los recursos para el desarrollo del organismo que en el proceso de su gestión busca lograr un cambio planeado en la entidad acorde con las exigencias demandas o necesidades de los diferentes sectores de la sociedad y de la misma institución, constituyéndose en el componente clave mediante la gestión en busca del cambio con el propósito de lograr un mejor desempeño y mayor eficiencia en la entidad, requisito indispensable para el avance de la institución en el mundo actual.

El sistema de salud del Ecuador se caracteriza por ser un sistema mixto, por el fraccionamiento por cuanto posee instituciones en el sector público las mismas que se desempeñan como privadas y que son financiadas por cotizaciones de los trabajadores del sector formal o por los aportes de grupos sociales de clase privilegiada circunscritos en una labor especifica, simpatizando con sistemas privados para la población de mayor poder adquisitivo, con actuaciones de salud pública y con servicios asistenciales para los más pobres; su actuación con bastante pesadumbre aparentemente claro en el ejercicio de su facultad, si bien la ley proclama la cobertura universal en salud, en los hechos todavía existe una limitada cobertura real con servicios cuya calidad no siempre es la óptima. En a práctica esta limitado a otras funciones como en el caso de supervisión, ante la autonomía y aparente independencia que muestran las instituciones que conformas el sistema. 
Los suministradores y financieros de salud que son algunos entre ellos el Seguro Social IESS, el mismo Ministerio de Salud Pública, ONG's, etc., que desarrollan actividades independientemente, el Sistema de Seguridad Social no tiene como objetivo la prestación de servicios de salud sino, únicamente, la de brindar un seguro que permita a sus afiliados acceder a estos servicios cuando lo requieran. Los servicios de salud, por su lado, son parte del Sistema Nacional de Salud. Si bien se contemplan las prestaciones de servicios de salud, así como la universalización de la atención, lo que puede generar confusiones. Sin embargo, el Sistema de Seguridad Social no tiene como objetivo la prestación de servicios de salud sino, únicamente, la de brindar un seguro que permita a sus afiliados acceder a estos servicios cuando lo requieran. La cobertura de la seguridad social es relativamente baja (IESS $10 \%$ y Seguro Campesino 10\%) y la red asistencial muy limitada quedando aproximadamente sin cobertura un 30\% de la población.

La administración de recursos humanos sirve para mantener la organización productiva, eficiente y eficaz, a partir del uso adecuado de su recurso humano. Es un avance percibir que las personas que son la fuerza del trabajo en la institución representan el recurso crítico, o sea, es aquel que sin el cual, la entidad no consigue realizar su trabajo.

\section{Referencias Bibliográficas.}

Amick, B., S. Levine, Tarlov, A., Walsh. D., (1995), Societies and Health. New York: Oxford University Press.

ARCSA, (s/f), Objetivos estratégicos y estrategias: Ejes Estratégicos, Agencia Nacional de Regulación, Control y $\quad$ Vigilancia $\quad$ Sanitaria (ARCSA), https://www.controlsanitario.gob.ec/ejesestrategicos/

Arriagada, I., Aranda, V. y Miranda, F., (2005), Políticas y programas de salud en América Latina. Problemas y propuestas, División de Desarrollo Social de la Comisión Económica para 
América Latina y el Caribe (CEPAL), ISSN electrónico 1680-8983, Naciones Unidas, Santiago de Chile.

CONASA, (2008), Informe de consultoría 2008, (elaborado por Alba Jorge), Consejo Nacional de Salud, Quito, Ecuador

CONEA, (2011), Consejo de Evaluación, Acreditación y Aseguramiento de la Calidad de la Educación Superior del Ecuador, Quito.

Constitución del Ecuador, (2008). Constitución política de la República del Ecuador, 2008, Título VII, Régimen del Buen Vivir, Capítulo Primero, Sección segunda, Salud, (Art. 362, 363, 369), Registro Oficial No 1,19 de octubre.

Espinosa V, Acuña C, De la Torre D, Tambini G., (2017), La reforma en salud del Ecuador. Rev Panam., Salud Pública. 41:e96.

Espinosa, V., de la Torre, D., Acuña, C. y Cadena, C., (2017), Los recursos humanos en salud según el nuevo modelo de atención en Ecuador. Rev Panam Salud Pública, 41:e52.

García, B. M., (2006), Gerencia de procesos para la organización y el control interno de empresas de salud, Ecoe ediciones, Bogotá.

Hermida, J., Romero, P., Abarca, X., Vaca, L., Robalino, M. y Vieira L., (2005), La Ley de Maternidad Gratuita y Atención a la Infancia en el Ecuador. Informe LACRSS: 63.

IESS, (s/f), Instituto Ecuatoriano de Seguridad Social. Seguro de Salud Disponible en: http://www.iess.gov.ec/site.php?category=seguro-de-salud.

Ley de Seguridad Social de las Fuerzas Armadas, (1992), Registro Oficial del 7 de agosto de 1992: 995.

Ley de Seguridad Social de la Policía Nacional, (1995), Registro Oficial del $1^{\circ}$ de junio de 1995 : 707.

Ley 55, (2001), Ley de Seguridad Social, Registro Oficial del 30 de noviembre de 2001: 465. 
IESS, (2010), Registros institucionales 2010. Instituto Ecuatoriano de Seguridad Social, Quito.

INEC, (2007), Encuesta de Recursos y Actividades en Salud: Instituto Nacional de Estadística y Censos, Quito.

ISAGS, (2012), Sistema de Salud en Ecuador, Instituto Suramericano de Gobierno en Salud, Rio de Janeiro.

ISSFA, (2010), Registros institucionales 2010, Instituto de Seguridad Social de las Fuerzas Armadas (ISSFA), Quito.

LMG, (1994), Ley de Maternidad Gratuita y Atención a la Infancia. Registro Oficial 1994; 9 de septiembre: 523 .

LOSEP, (2010), Ley Orgánica de Servicio Público, Asamblea Nacional, Quito.

Lucio, R., Villacrés, N. y Henríquez, R., (2011), Sistema de salud de Ecuador, Ministerio de Coordinación de Desarrollo Social, Quito, Ecuador.

Lucio, R., (2010), Modelo de salud en Ecuador, Ministerio Coordinador de Desarrollo Social, Quito.

Lucio, R., (2007), La salud en Quito: análisis de acceso y calidad, Cooperación Técnica Belga en Ecuador, Quito.

Malo S., M. y Malo C., N., (1014), Reforma de salud en Ecuador: nunca más el derecho a la salud como un privilegio. Rev Perú Med Exp Salud Pública, 31(4):754-61.

MDT y MSP, (2015), Norma Técnica para la Implementación del Internado Rotativo en los establecimientos de salud de la Red Pública Integral de Salud, Ministerio del Trabajo, Ministerio de Salud Pública.

MCDS, (2011), Ministerio de Coordinación del Desarrollo Social: MCDS, Ecuador.

Ministerio de Relaciones Laborales, (2012), Norma técnica para el reconocimiento de la bonificación geográfica a las y los servidores del sector de salud pública. Quito.

Mintzberg, H., Quinn B., J., (1993), “El proceso estratégico: Conceptos, Contextos y Casos”. P.5. 
Naciones Unidas (2005), Objetivos del Milenio. Una mirada desde América Latina y el Caribe LG.2331, Santiago de Chile.

Nigenda, G., Troncoso, E., Arreola, H., López, M., y González, L., (s/f), Mezcla público-privada en el sector salud. Reflexiones sobre la situación en México, Innovaciones en salud.

OPS, (2008), La formación en medicina orientada hacia la atención primaria de salud, Organización Panamericana de la Salud, Washington, DC.

OPS, (2007), Estrategias para Desarrollar las Competencias para la Salud Pública en la Fuerza de Trabajo: Basadas en Políticas Actuales y en Evidencias, Organización Panamericana de la Salud, ISBN 978-92-7-532835-4, (USAID).

OPS, (2005), La enfermería de salud pública y las funciones esenciales de salud pública: bases para el ejercicio profesional en el siglo XXI. Biblioteca Lascasas; 1. Disponible en http://www.indexf.com/lascasas/documentos/lc0054.php

OPS, (2001), En la mayor evaluación internacional realizada hasta la fecha, los Centros para el Control de Enfermedades (CDC) colaboraron con la Organización Panamericana de la Salud para ayudar a 2.000 trabajadores de salud de 41 países de América Latina y el Caribe a realizar autoevaluaciones de sus sistemas de salud pública. Citado por OPS, (2007).

OPS, (2000), Estrategia para el fortalecimiento de la promoción de la salud en los lugares de trabajo en América Latina y el Caribe, Organización Panamericana de la Salud, San José, Costa Rica.

Ponce A., Jhon, (2015), Sistema de Salud del Ecuador y la Atención Primaria de la Salud, Tesis de posgrado Doctorado en Salud Pública, Universidad de ciencias empresariales y sociales, Buenos Aires - Argentina.

Rosenberg, M.W. y Lovell, S., (2006), "A Methodology for Projects to Characterize the Public Health Workforce in Costa Rica, Jamaica, and México”, Departamento de Geografía de la Universidad Queen, Kingston, Ontario, Canadá. 
Juan M. Haro-Alvarado; Javier I. Haro-Alvarado; Mariela G. Macías-Intriago; Nakin A. Veliz-Mero; Julissa F. ToalaSornoza; Tatiana A. Solís-Lino

Regás P., Ibern, (2009), Interacciones público-privadas en el ámbito de la salud, Rev Adm Sanit.; $7(1): 33-42$

Superintendencia de Bancos y Seguros del Ecuador, (s/f), Seguridad Social: Entidades Controladas, Disponible en: http://www.superban.gov.ec/practg/sbs_indexivp_art_id=46\&vp_tip=2.

Valencia Díaz, Andrés A., (2015), Estrategia de la administración, 328, https://prezi.com/f2c9v9h9zcr7/estrategias-de-la-administracion/

Zaratiegui, J. R., (1999). La gestión por procesos, Economía industrial, 81-88. 